\title{
Pros y contras de las innovaciones en educación médica
}

\author{
Alberto Lifshitz-Guinzberg, ${ }^{1,2 *}$ Luis F. Abreu-Hernández, ${ }^{1,3}$ Ana C. Sepúlveda-Vildósola, ${ }^{1,4}$ \\ Ma. Esther Urrutia-Aguilar, ${ }^{1,5}$ José Á. Córdova-Villalobos, ${ }^{1,6}$ Joaquín López-Bárcena ${ }^{1,7}$ y \\ Melchor Sánchez-Mendiola²
}

${ }^{1}$ Academia Nacional de Medicina, Comité de Educación Médica; 2Universidad Nacional Autónoma de México, Facultad de Medicina; ${ }^{3}$ Universidad Nacional Autónoma de México, División de Estudios de Posgrado; ${ }^{4}$ Instituto Mexicano del Seguro Social, Coordinación de Investigación en Salud; ${ }^{5}$ Universidad Nacional Autónoma de México, Unidad de Posgrado; ${ }^{6}$ Exsecretario de Salud de México; ${ }^{7}$ Academia Nacional de Educación Médica. Ciudad de México, México

\section{Resumen}

La educación médica ha puesto en práctica diversas estrategias innovadoras con el propósito de alcanzar mejores logros de aprendizaje. Se hace una evaluación de las experiencias relacionadas con el enfoque por competencias, las nuevas tecnologías educativas, las alternativas curriculares, la profesionalización de la evaluación y las técnicas educativas a distancia, para ubicarlas en el lugar que les corresponde.

PALABRAS CLAVE: Educación. Tecnología educativa. Competencias. Evaluación. Currículo.

\section{Pros and cons of innovations in medical education}

\begin{abstract}
Medical education has implemented various innovative strategies with the purpose to attain better learning achievements. An evaluation is made of the experiences in the competencies approach, new learning technologies, curricular alternatives, professional evaluation and distance education technologies in order to locate them in the areas they belong.
\end{abstract}

KEY WORDS: Education. Learning technology. Competencies. Evaluation. Curriculum.

\section{Introducción}

\section{Alberto Lifshitz}

La innovación y la creatividad son dos valores muy bien ponderados por la sociedad y son el fundamento del progreso. La educación médica tiene un compromiso con los aprendices para lograr mayor eficiencia en el aprendizaje y, por ello, está en una constante búsqueda de mejores estrategias. Las circunstancias actuales han propiciado una aceleración de esta búsqueda, pero siempre es conveniente una llamada de alerta en el sentido de que no necesariamente lo más nuevo es lo mejor, al menos no únicamente por ser nuevo. Así han surgido no solo innovaciones sino simples novedades, modas, ocurrencias, caprichos, cambios insustanciales o hasta menoscabos que aparentan ser progresos. Por ello, siempre conviene una postura crítica sin deslumbramientos y, en cuanto sea posible, una evaluación de sus ventajas y desventajas. Las primeras impresiones no son siempre confiables y se tiene que dar tiempo a que se pongan a prueba.
Correspondencia:

*Alberto Lifshitz-Guinzberg

E-mail: alifshitzg@yahoo.com

DOI: 10.24875/GMM.20000688
Fecha de recepción: 19-09-2020

Fecha de aceptación: 10-01-2021

DOI: $10.24875 /$ GMM.20000688
Gac Med Mex. 2021:157:338-348

Disponible en PubMed

www.gacetamedicademexico.com CC BY-NC-ND (http://creativecommons.org/licenses/by-nc-nd/4.0/). 
Algunas de las innovaciones que aquí se mencionan pudieran parecer no tan nuevas, pero es un buen momento para someterlas a un análisis crítico.

\section{Educación por competencias}

\section{Luis F. Abreu-Hernández}

Los médicos competentes son aquellos que poseen el potencial para cumplir eficientemente con las funciones demandadas a la profesión por la sociedad; muestran su capacidad de actuar en los ambientes dinámicos y complejos en los cuales se ejerce la medicina, lo que implica la articulación de conocimiento, procesos cognitivos y habilidades intelectuales, destrezas, valores, cultura y relaciones sociales, que permiten dar respuesta a los requerimientos de la práctica profesional presente y futura y conservan la calidad del ejercicio en una pluralidad de contextos y situaciones. Mantienen la capacidad de establecer cursos de acción óptimos mediante la evolución constante, la capacidad para trabajar en equipo, la reflexión en la acción, el conocimiento científico, el manejo multidisciplinario, la flexibilidad, la adaptabilidad, la evaluación de resultados, el compromiso social y la visión de futuro.

El sociólogo Eliot Freidson ${ }^{1}$ describió a la medicina como una profesión caracterizada por el ejercicio de la autonomía, en la cual los médicos toman decisiones para resolver los problemas de salud, basados en el corpus de conocimiento médico. Frente a esta noción abierta y dinámica, se ha desarrollado otra de carácter burocrático tendente a limitar a autonomía profesional mediante la aplicación de métodos de administración industrial. Se ha pretendido controlar el actuar de los médicos, anteponiendo los ahorros y las ganancias a la atención a la salud y a la empatía con el paciente, se ha intentado convertir a los médicos en seres instrumentales que aplican normas, guías, protocolos y procesos estandarizados de manera acrítica, bajo el supuesto de que todos los pacientes son iguales y responden de la misma forma. La atención a la salud se ha transformado en un sistema industrial que procesa individuos de manera mecánica, deshumanizada, con prioridades ajenas a la salud de las personas y la cual ha sido caracterizada como "medicina industrializada". ${ }^{2}$

Esta polarización se ha traducido en dos definiciones confrontadas de las competencias. Barton Childs ${ }^{3}$ ha señalado que la medicina no puede sustentarse en la creencia de que todos los pacientes son iguales y responden de la misma forma, porque la medicina tiene que reconocer la variabilidad genética y la variabilidad social y que cada paciente es único. Por ello, el médico debe contextualizar el saber a cada paciente concreto, lo cual se ha denominado "medicina de precisión". ${ }^{4}$ Una aplicación a rajatabla de la medicina basada en la evidencia conduce al error e impide los avances. Donald Berwick, uno de los mejores expertos en calidad de la atención, ha señalado que al eliminar las variaciones también ignoramos el contexto y ha afirmado que "sin evidencia podemos hacer daño, pero también es igualmente dañino tratar a un fenómeno complejo como si fuera simple y cuando tratamos a las personas únicas como si fueran partes simples y separadas de nosotros, reducimos nuestra capacidad de ver aprender, conversar y crecer". ${ }^{5}$

Resulta conveniente distinguir entre la competencia real de los profesionales de la medicina vivos y actuantes y la competencia como descripción escrita, destinada a orientar las actividades de un plan de estudios; la segunda se obtiene en parte de la primera, pero son diferentes entre sí. Frecuentemente se confunden las competencias con las actividades: colocar una sonda nasogástrica, poner un catéter venoso o colocar una férula son actividades y carecen de valor en sí mismas, porque solo adquieren significado cuando se encadenan con un propósito determinado. Las competencias consisten en la descripción de los grandes propósitos de la profesión, equivalentes a las grandes funciones profesionales que dan un sentido al todo el quehacer profesional. Para redactar las competencias hay que analizar a los practicantes prototípicos de la profesión mediante diversas técnicas que se conocen como análisis de tareas, ${ }^{6}$ que consisten en entrevistas, consenso de expertos, estudios de sombra, caminar y hablar, incidente crítico y

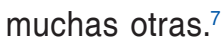

No obstante, si únicamente tomásemos como modelo a los profesionales actuales, estaríamos suponiendo que el futuro será igual al presente, afirmación insostenible en un campo tan dinámico como la medicina; hay que añadir un enfoque prospectivo para determinar qué cambios visualizamos en el horizonte de la profesión. Si la descripción es muy precisa, la competencia se vuelve rígida y no permitirá asimilar los cambios y si es muy general, no permitirá orientar al proceso formativo; generalmente se requiere lograr un justo medio. Debido a lo anterior, no es fácil redactar competencias. Un consejo práctico para quienes se inician en esta tarea es responder las siguientes preguntas para cada competencia: 
¿qué va a hacer el profesional (verbo)?, ¿para qué lo va a hacer (propósito)?, ¿en qué contexto lo va a realizar (situación)?, ¿cómo lo debe hacer y qué debe evitar (criterios de ejecución)? Este último rubro va dirigido a orientar la evaluación. Las competencias siempre serán afirmaciones abiertas y un tanto cuanto imprecisas para permitir el cambio, por ello han sido descritas como fuzzy learning outcomes. ${ }^{8}$

El propio Childs $^{3}$ ha distinguido dos enfoques educativos: la instrucción, denominada así porque enseña a seguir instructivos; y la formación, que enseña a evolucionar continuamente, desarrolla el pensamiento crítico y nos prepara para adecuarnos al contexto y avanzar continuamente.

En la medicina actual no podemos formar "médicos obreros" seguidores de instructivos que aplican rutinas de manera mecánica, acrítica e insensible, y que primordialmente sirven a las instituciones de salud deformadas y secundariamente a las personas.

El proceso formativo orientado por competencias no se obtiene mediante las simple suma de materias aisladas que milagrosamente se integran y producen un médico adaptativo; implica una planeación de arriba hacia abajo, en la cual todo el currículo está alineado con las competencias finales y todas las materias realizan su aportes. Exige transitar de la formación de repetidores de instrucciones hacia la formación de "navegantes", capaces de asumir nuevos retos y seguir el avance continuado del conocimiento. A tal fin, el proceso educativo dinámico es el contenido más relevante del curso. ${ }^{9}$ No se trata solo de arribar al resultado correcto, sino también desarrollar procesos correctos.

\section{Innovaciones tecnológicas}

\section{Ana Carolina Sepúlveda-Vildósola}

La innovación puede limitarse a cambios que afectan parcialmente alguna de las prácticas existentes (mejora continua), al refinamiento y mejora de algún elemento, metodología, estrategia, proceso, medio de entrega o procedimiento (incremental); a aplicar un nuevo paradigma que cambia significativamente las prácticas existentes (revolucionaria) o representa un cambio drástico en dicha práctica (disruptivo). ${ }^{10} \mathrm{El}$ paradigma de atención médica centrada en individuos, su tratamiento y la rehabilitación del daño ha evolucionado a uno en donde el control de las determinantes sociales de la enfermedad, la prevención y la participación activa de las personas en la promoción a la salud y el tratamiento de sus enfermedades cobran cada vez mayor relevancia. En la actualidad, los avances tecnológicos en salud permiten ofrecer tratamientos personalizados, predictivos y de precisión en contraste con los tratamientos estándar del pasado.

De la misma forma, los paradigmas educativos han evolucionado, de los modelos pedagógicos pasivos y el aprendizaje memorístico a la participación activa del alumno, la reflexión y la construcción colectiva del conocimiento. Los modelos de educación masificada ahora están centrados en el alumno, permiten currículos flexibles y personalizados, que consideran los intereses del educando. La innovación tecnológica (por ejemplo, la simulación) ha facilitado la práctica deliberada y la adquisición de competencias de una forma segura, tanto para el paciente como para el alumno.

Prácticamente todos los estudiantes de medicina cuentan en la actualidad con un teléfono o tableta inteligente, que son computadoras de bolsillo con diversidad de funciones que favorecen y facilitan la educación. ${ }^{11,12}$

Las prácticas educativas en el aula se han facilitado con la introducción de tecnología como los pizarrones inteligentes y los videoproyectores, cada vez más compactos y de mejor resolución. La gamificación en la instrucción (por ejemplo, Kahoot) favorece la atención y participación activa de los alumnos. El uso de redes sociales en el proceso educativo favorece la comunicación, el trabajo en equipo y la retroalimentación, entre otros. ${ }^{13}$

La disponibilidad de materiales educativos en formatos digitales (por ejemplo, en discos compactos, aplicaciones o sitios electrónicos), así como de cursos a distancia, blogs y webinars en plataformas en el ciberespacio, permite un mayor acceso e individualización de la educación de acuerdo con los intereses personales y favorece la educación continuada, disminuyendo costos y barreras geográficas. ${ }^{14}$

La simulación ha cobrado gran importancia desde hace algunas décadas. La recreación de escenarios reales, mediante tecnología de baja o alta fidelidad o realidad virtual, permite al alumno adquirir competencias diversas. La inteligencia artificial se ha incorporado tanto al proceso de atención médica, como a la educación en salud; permite automatizar tareas, predecir, acompañar y evaluar. ${ }^{15,16}$

La tecnología debe verse como un medio y no como un fin de la educación. Ofrece innumerables ventajas, pero no está exenta de desventajas. ${ }^{17}$ La tabla 1 
Tabla 1. Ventajas y desventajas

\begin{tabular}{|c|c|}
\hline Ventajas & Desventajas \\
\hline - Aceptabilidad y frecuencia de uso & $\begin{array}{l}\text { - Tiempo y costo } \\
\text { de materiales y/o } \\
\text { adquisición del equipo }\end{array}$ \\
\hline $\begin{array}{l}\text { - Favorece el aprendizaje activo } \\
\text { y colaborativo, la colaboración, } \\
\text { la inmersión al contenido, la } \\
\text { interacción, el compromiso, la } \\
\text { construcción de experiencias, } \\
\text { la autoeficacia y el aprendizaje } \\
\text { contextualizado socialmente }\end{array}$ & $\begin{array}{l}\text { - Calidad variable. Debe } \\
\text { integrar componentes } \\
\text { médico, educativos y } \\
\text { tecnológicos }\end{array}$ \\
\hline $\begin{array}{l}\text { - Favorece acceso y autogestión del } \\
\text { aprendizaje (life-long-learning) }\end{array}$ & - Barrera generacional \\
\hline $\begin{array}{l}\text { - Facilita la conexión entre } \\
\text { aprendizaje formal e informal } \\
\text { - Ambientes seguros (paciente y } \\
\text { alumno) }\end{array}$ & $\begin{array}{l}\text { - Resistencia al cambio/ } \\
\text { ideas preconcebidas }\end{array}$ \\
\hline $\begin{array}{l}\text { - Educación personalizada, global } \\
\text { - Recrea situaciones poco } \\
\text { frecuentes }\end{array}$ & $\begin{array}{l}\text { - Falta de motivación } \\
\text { - Requiere capacitación y } \\
\text { habilidades tecnológicas }\end{array}$ \\
\hline $\begin{array}{l}\text { - Multiplataforma y puede usarse } \\
\text { para convertir el tiempo de ocio en } \\
\text { productivo } \\
\text { - Experiencias escalables } \\
\text { - Se puede repetir hasta lograr la } \\
\text { competencia }\end{array}$ & $\begin{array}{l}\text { - Creatividad para su uso } \\
\text { como recurso educativo } \\
\text { - Uso no profesional del } \\
\text { recurso }\end{array}$ \\
\hline $\begin{array}{l}\text { - Favorece la sana competencia } \\
\text { entre alumnos y la transferencia a } \\
\text { situaciones reales. } \\
\text { - Mayor satisfacción, aprendizaje } \\
\text { disfrutable, mayor motivación; al } \\
\text { final, sensación de ganancia } \\
\text { - Permite tener información en } \\
\text { tiempo real, en la cama del } \\
\text { paciente } \\
\text { - Permite llevar historial } \\
\text { individualizado de avances }\end{array}$ & $\begin{array}{l}\text { - Recursos disponibles } \\
\text { - Apoyo institucional } \\
\text { - Aumento de la carga } \\
\text { docente } \\
\text { - Difícil la evaluación } \\
\text { objetiva del alumno } \\
\text { - Privacidad } \\
\text { - Propiedad intelectual del } \\
\text { material } \\
\text { - Variabilidad en la } \\
\text { participación de los } \\
\text { estudiantes }\end{array}$ \\
\hline
\end{tabular}

resume los pros y contras de la tecnología en la educación en salud.

En los últimos meses, la pandemia de COVID-19 ha acelerado el uso de la tecnología en la educación. Al suspenderse las actividades educativas presenciales, se iniciaron clases a distancia mediante plataformas en tiempo real. Esta fue una solución a corto plazo y debemos prepararnos para potencializar el uso de las tecnologías de la información y la comunicación a mediano y largo plazo, aprovechando la transformación digital. La gamificación parte de la premisa de que "se aprende mejor jugando" y aprovecha la inclinación del ser humano hacia la competición y el entretenimiento. Consiste en el desarrollo de juegos que permiten al individuo aprender a partir de la resolución de problemas o retos y superar etapas con distintos grados de complejidad digital, ${ }^{18}$ para lograr una innovación disruptiva en el proceso.

\section{Innovación curricular}

\section{María Esther Urrutia-Aguilar}

El concepto de innovación está relacionado con la creación de algo desconocido, la percepción de lo creado como algo nuevo y la asimilación de ese algo como novedoso. ${ }^{19}$ Según Elmore, ${ }^{20}$ en la innovación educativa podemos distinguir entre cambios estructurales, que afectan a todo el sistema educativo, o los que afectan a la configuración de los distintos niveles; cambios curriculares, relacionados con el diseño y desarrollo; cambios en los materiales curriculares, con nuevos enfoques de enseñanza; cambios profesionales referidos a la formación, selección y desarrollo profesional de los docentes; y cambios políticos y sociales, que afectan a la distribución del poder en la educación y la relación de los agentes sociales con la enseñanza escolar.

Este escrito solo se referirá a la innovación curricular que cita Beltrán, ${ }^{21}$ quien considera que el currículo no es un objeto, sino un proceso que debe incluir las opiniones de los docentes. Elliott ${ }^{22}$ señala que el profesorado es un agente de cambio en las situaciones educativas.

A partir del siglo pasado han existido tres reformas en la educación médica: la primera a principios del siglo $X X$, en la que los planes de estudio se estructuraron con base en la ciencia; la segunda a mediados del mismo siglo, la cual introdujo innovaciones educativas como el aprendizaje basado en problemas y la medicina basada en la evidencia; actualmente se vive la tercera reforma, basada en la mejora del rendimiento de los sistemas de salud mediante la adaptación de las competencias profesionales básicas a contextos específicos.

A principios del siglo pasado se consideraba la formación médica en dos etapas: la primera enfocada en el estudio de las ciencias básicas y la segunda, en la práctica clínica en hospitales. A mediados de ese siglo se inició una etapa de cuestionamientos a la estructura de los planes de estudios, a su rigidez curricular, al enfoque centrado en la enseñanza y a la falta de una visión integral del paciente en su dimensión biopsicosocial. ${ }^{23}$ Además, las políticas 
educativas estuvieron marcadas por la globalización, los tratados de colaboración y comercio internacional, la búsqueda de la certificación y homologación de programas y profesiones y la definición de estándares nacionales e internacionales. ${ }^{24}$

A mediados del siglo pasado se introdujeron como innovación curricular enseñar a los estudiantes a utilizar la mejor evidencia científica disponible para la toma de decisiones, la incorporación de la medicina basada en evidencias y el aprendizaje basado en problemas. Asimismo, se incluyeron también las teorías de liderazgo y la informática biomédica, esta última orientada a desarrollar las capacidades del médico para manejar la información. ${ }^{25}$

Actualmente, la mayoría de las escuelas están completamente enfocadas en la educación de primera generación, con planes de estudio y métodos de enseñanza tradicionales y estancados, con incapacidad o, incluso, resistencia para cambiar, lo cual no ayuda a la formación de los médicos que se necesitan, debido a que prevalecen planes de estudios fragmentados, obsoletos y estáticos. ${ }^{26}$ Sus egresados se gradúan con desajustes en las competencias para resolver las necesidades de los pacientes y de la población, con una pobre formación para el trabajo en equipo y con tendencia a actuar de forma aislada. Algunos países han incorporado reformas de segunda generación y unos pocos se están mudando a la tercera generación.

Actualmente existe un vertiginoso desarrollo de las tecnologías como la robótica, los grandes datos y la inteligencia artificial; a ello se suman los cambios sociales resultado de la globalización y de las migraciones, de la aparición de nuevas enfermedades, de los nuevos riesgos infecciosos, ambientales y de comportamiento, de los cambios en la demografía, de las aportaciones transdisciplinarias que han sustituido a las disciplinarias y de que los pacientes están más informados hoy en día.

Los beneficios de todos esos desafíos deberán ser orientados a una mejor atención médica, a promover la educación interprofesional y transprofesional; para ello, es necesario explotar los recursos digitales y una medicina más humanista. El objetivo deberá ser el aprendizaje de los estudiantes para recuperar, seleccionar, evaluar y priorizar la información, desarrollar el razonamiento crítico y adquirir conductas éticas, pero, sobre todo, integrarse como miembros de equipos receptivos y conectados globalmente.

Otro de los aspectos importantes por considerar es el aumento explosivo del volumen de la información y la facilidad de acceso a ella, por lo que la próxima generación de alumnos necesita desarrollar la capacidad de discriminar grandes cantidades de información y extraer y sintetizar el conocimiento que sea necesario para las decisiones clínicas. ${ }^{27}$

\section{Innovación curricular parte II}

\section{José Ángel Córdova Villalobos}

\section{En 2013, la American Medical Association propuso} un pilar de la educación médica llamado

Ciencias de Sistemas de Salud (Health System Sciences), el cual comprende entre otros temas:

- Seguridad del paciente: un tema fundamental cuya importancia se ha fortalecido a raíz de los informes relacionados con los errores médicos. En consecuencia, movimientos como Patient Safety, en más de 40 países han diseñado 16 apps que recuerdan las acciones médicas para evitar accidentes o evolución inadecuada.

- Mejora de la calidad de atención: buscar acceso a la salud para todas las personas por el solo hecho de ser humanos. Pero esta prerrogativa sería incompleta si el acceso no se complementa con la calidad de la atención, para lo que se requiere personal capacitado, no solo técnicamente, sino con actitud de servicio, instalaciones, equipos e insumos necesarios.

- Medicina basada en evidencias: constituye el mayor respaldo científico para el profesional de la salud para lograr los mejores resultados en los pacientes.

- Valor de los servicios de salud: que los tomadores de decisiones financieras entiendan que la salud "no es un gasto, sino una inversión" y que si no se cuenta con un apoyo sólido en cuanto a instalaciones, materiales, equipo y medicamentos, no basta la vocación, la dedicación, los conocimientos y las actitudes del personal de salud para obtener buenos resultados.

- Trabajo interdisciplinario: hoy en día, los equipos de salud no son solamente médicos (generales o especialistas), sino que el trabajo de enfermeras, nutricionistas, químicos, rehabilitadores, odontólogos, psicólogos, trabajadores sociales, ingenieros biomédicos y sanitaristas es igualmente importante.

- Coordinación del cuidado de la salud: es fundamental la distribución y acatamiento de las funciones de cada uno de los miembros del equipo 
de salud; "nadie es más que nadie, todos son importantes"

- Gestión de recursos: en la mayoría de las escuelas de medicina no se enseña la forma de cómo y ante quién gestionar los recursos para el buen funcionamiento del sistema, la institución o la unidad; estos aspectos son más bien terreno de una especialidad, pues el médico debe conocer de planeación, administración y gestión de recursos para estar preparado en cualquier momento para ocupar un puesto directivo.

- Administración de poblaciones: tanto en la medicina de atención primaria, como en la gestión de la salud pública, es muy importante para implementar la mayoría de los programas preventivos y curativos.

- Informática biomédica: la capacitación en conocimientos de informática es indispensable desde la formación escolar, primaria, secundaria y preparatoria, y debe ser la base para facilitar la búsqueda permanente de información al futuro médico, para aumentar su acervo y, por supuesto, resolver problemas.

- Liderazgo: no solo en medicina sino en cualquier profesión o trabajo y en la vida diaria es fundamental aprender a ser líderes o desarrollarlo para motivar los cambios, acelerar los avances y generar modelos.

- Financiamiento: generar la conciencia de que la salud es una inversión, para cumplir con el segundo derecho humano más importante después del derecho a la vida, con base en una buena planeación y un correcto uso de los recursos.

- Reforma de los servicios de salud: hemos vivido reformas energéticas, educativas, hacendarias, etcétera; sin embargo, la reforma a la salud no se ha dado, ni en los servicios para fortalecer la atención primaria ni en la formación de recursos humanos, pues los currículos en muchas instituciones están enfocados a preparar egresados capaces de ser seleccionados para continuar con una especialidad y no con la visión de primero ser muy buenos médicos generales. Es necesario trabajar en niveles anteriores (la preparatoria o la secundaria) para acostumbrar a los alumnos a mayor trabajo coordinado en equipo a nivel de la comunidad, para que desde ahí reconozcan, vaIoren y trabajen en la educación, la participación y el liderazgo; para que al llegar a la universidad se acostumbren a trabajar en equipo, a respetar el valor de cada uno de sus compañeros, a diseñar proyectos y llevarlos a cabo en las comunidades más necesitadas, con apoyo de sus maestros y la participación de especialistas en los centros de atención primaria.

Se requiere también de trabajo interprofesional, entre diferentes elementos del sistema de salud y con otras personas de diferentes profesiones, trabajando en equipo.

El replanteamiento del nuevo currículo deberá tomar en cuenta el sistema educativo, los requerimientos del sistema de salud de acuerdo con la reforma, la demanda de cierto tipo de personal sanitario (médicos de atención primaria), las características y necesidades de la población y el mercado laboral. Asimismo, deberá tratarse de un currículo dinámico en el que se tomen en cuenta los valores, los roles, la comunicación, el trabajo en equipo y con el cual el alumno vaya teniendo un aprendizaje activo.

\section{En resumen:}

- Se requiere de un diálogo intenso y sostenido entre las universidades formadoras de recursos humanos en salud y el sector salud, que constituya una reflexión crítica que permita crear las bases para un desarrollo profesional y un cambio dinámico, sostenible y profundo.

- A cada paso habrá que investigar los riesgos y las oportunidades que cada innovación curricular lleva aparejada.

- Será necesario rescatar el protagonismo del profesorado en los procesos de desarrollo curricular, dado que las innovaciones suscitan controversias porque cuestionan creencias sobre la enseñanza, el aprendizaje y la evaluación, debido a una resistencia al cambio.

- Se requiere enfocar el papel del médico como educador, incorporar habilidades de tipo administrativo y de gestión, así como agregar un eje de investigación que prepare al estudiante en el manejo de la información, las tecnologías de la información y comunicación, la informática médica y la metodología científica, sin marginar los componentes éticos y humanitarios.

- La participación de los alumnos será fundamental en el enriquecimiento a través de las vivencias y experiencias, así como la evaluación de las innovaciones, dentro de un currículo flexible y dinámico que siempre buscará ser mejor. 


\section{Innovación en evaluación}

\section{Joaquín J. López Bárcena}

Algunas propuestas permiten identificar cambios que generan un nuevo paradigma que repercute en las prácticas existentes (revolucionarios), otros que mejoran alguno de los componentes del proceso educativo (incrementales), o bien cambios que son reproducidos de otros modelos y que son poco relevantes (de mejora continua). ${ }^{28}$

Si se entiende la evaluación como un proceso que permite obtener información para tomar decisiones, queda claro que no se puede llevar al cabo de manera aislada y que es aplicable a todos los componentes de un programa educativo. Así, para evaluar es necesario contestar las preguntas ¿qué?, ¿para qué? y ¿cómo? Las innovaciones quedan vinculadas a las que se incorporan al planteamiento educativo. 29,30

Las innovaciones se pueden ubicar en el diseño curricular, en el método educativo y en la modalidad instruccional. Desde Flexner, en 1910, hubo una inercia hasta la década de 1960 cuando se encontró alguna uniformidad sobre lo que se tenía que evaluar: ¡los objetivos!, con un nivel de impacto prácticamente universal. Los instrumentos más utilizados fueron las pruebas escritas estructuradas, con predominio de cuestionarios con opciones múltiples de respuesta, muy útil para grupos grandes, pero limitada a un ejercicio memorístico y al azar. Esto prevalece hasta la actualidad.

La siguiente innovación ocurrió al inicio del presente siglo, con la aparición de la educación basada en competencias, ${ }^{31}$ entendidas como un conjunto de dominios (saberes) que se aprenden en forma simultánea y de manera progresiva. Incluyen los conocimientos, las habilidades y destrezas, el comportamiento, la convivencia y el trabajo en equipo, el aprendizaje autorregulado y autónomo, así como la creatividad y el emprendimiento.

En años recientes ha surgido otra novedad educativa, que complementa las competencias; se trata de las "actividades profesionales confiables" (APROC), versión latina de las entrustable professional activities (EPA), generadas en Holanda y que para su evaluación ha requerido la creación de aplicaciones digitales para dispositivos móviles, que permitan al profesor realizarla durante el desempeño clínico cotidiano. ${ }^{32-34}$
Todo esto implica que el proceso de evaluación tiene que ser polifacético, conservando la validez y la confiabilidad. Hasta la fecha, continuamos con las competencias y con la mejora de los nuevos instrumentos de evaluación, que también tienen que estar de acuerdo con las variantes del método educativo y las modalidades instruccionales. Esto significa que tienen que utilizarse varios instrumentos de evaluación para una sola competencia. De ahí que los cambios innovadores en evaluación han consistido en modificaciones a los cuestionarios de opción múltiple y las listas de cotejo, el uso de rúbricas, el uso de portafolios, los simuladores y, con todo su esplendor, los recursos de tecnologías de la información y de la comunicación (TIC), con la realidad virtual y la realidad aumentada, entre otras, así como, aunque aún en etapas iniciales, la robótica y telemedicina. ${ }^{35-37}$

En la década de 1980 se inició un cambio innovador que cimbró la metodología en la educación médica. Hasta entonces, las figuras principales en el proceso educativo eran el profesor y la enseñanza. El primero, como modelo a seguir, aunque predominó el profesor dogmático, autocrático, protagónico, autocomplaciente y resistente a cualquier cuestionamiento o evaluación sobre su proceder docente. El cambio ha sido drástico: ahora se priorizan el estudiante y el aprendizaje. El profesor se ubica en un segundo plano, como asesor, guía o facilitador y sujeto a constantes evaluaciones por los alumnos, las autoridades escolares y los pares. La enseñanza era memorística y enciclopedista; ahora se procura que el aprendizaje sea significativo, que se comprenda, que se logre explicar y que haya metacognición. También, que el estudiante aprenda a aprender, que desarrolle habilidades para alcanzar autonomía y pueda regular lo que necesita aprender; hoy ocupa el lugar que le corresponde: ser la esencia del proceso educativo y el motivo de existir del docente.

En paralelo, la penetración de las TIC y sus variantes, como las TICCAD (técnicas de información, comunicación, conocimiento y aprendizaje digital), ha favorecido la educación a distancia. Inicialmente utilizada para la educación continua y la capacitación, fue seguida de estudios escolarizados formales y actualmente, como lo hemos vivido recientemente por el confinamiento que exige la pandemia; ha sustituido a la educación presencial en todos sus niveles formativos. Si bien es cierto que debe ser temporal, se ha aprendido que habrá aspectos del aprendizaje que se continuarán con esta modalidad, debido a las ventajas que ofrece. 
Aunque en la educación médica, la educación a distancia todavía no es aplicable para varios elementos de aprendizaje clínico, han ocurrido innovaciones educativas con el uso de simuladores de diferentes niveles de complejidad. ${ }^{38,39}$ Para evaluar estos cambios es necesario actualizarse con el uso de estas novedosas herramientas educativas, porque con esas mismas habrá que diseñar los mecanismos de evaluación, de tal manera que se complementen los resultados obtenidos. Los cuestionarios de opción múltiple deben modificarse de tal forma que se requiera pensar para contestar, que se utilice el proceso de razonamiento clínico. ${ }^{40}$ Las listas de cotejo deben ser más flexibles y complementarse con las rúbricas. Pueden utilizarse representaciones gráficas (infografías, mapas conceptuales, diagramas de flujo, esquemas), que permitan expresar la comprensión de una situación clínica y su manejo, lo que simultáneamente permite evaluar el trabajo en equipo, la comunicación, la habilidad en la obtención y selección de la información, la creatividad en la expresión y presentación. Además, existe el recurso de emplear videos para demostrar procedimientos clínicos y quirúrgicos con simuladores, así como las habilidades clínicas para la entrevista con el paciente en diferentes circunstancias y el comportamiento con pares, superiores y subordinados.

El uso de varios instrumentos de evaluación y en diferentes momentos del proceso formativo requiere un registro evolutivo de los resultados obtenidos en cada uno, lo que favorece la autoevaluación reflexiva y la valoración integral del aprendizaje y, por otro lado, la creación constante de herramientas y estrategias que permitan la congruencia de lo que se quiere evaluar y cómo hacerlo. ${ }^{41,42}$

La innovación que ha tenido mayor impacto es el examen clínico, objetivo y estructurado (ECOE, traducción de OSCE, objective structured clinical examination), que si bien tiene cerca de tres décadas de haber iniciado, ha evolucionado. Posee una versatilidad que permite evaluar en forma simultánea varios componentes de una competencia, como la asimilación del conocimiento, la aplicación, comunicación humana, habilidad y destreza técnicas, trabajo en equipo, cuidado del paciente, manejo de la información y elementos conductuales en la relación médico-paciente. Requiere una variedad de escenarios clínicos, diversos instrumentos de evaluación, uso de la tecnología, manejo eficiente del tiempo, con la mayor objetividad posible. La grabación del desempeño en cada estación facilita la retroalimentación reflexiva, indispensable en todo proceso formativo; en realidad, más que un instrumento, es un formato que reúne varios instrumentos de evaluación. Un inconveniente es su alto costo, sobre todo si incorpora pacientes simulados estandarizados. ${ }^{43}$

La incorporación de nuevas tecnologías educativas implica el diseño innovador de instrumentos de evaluación con las mismas tecnologías. ${ }^{44,45}$

\section{Innovaciones emergentes}

\section{Melchor Sánchez-Mendiola}

La única manera de descubrir los límites de lo posible es ir más allá de ellos hacia lo imposible ARTHUR C. CLARKE

La pandemia ocasionada por el virus SAR-CoV-2 ha generado un impacto sin precedentes en la educación superior a nivel global, provocando un verdadero cataclismo. Ello ha provocado que los organismos internacionales dedicados a las políticas educativas, como la UNESCO, hayan establecido una serie de recomendaciones relevantes para las escuelas y facultades de medicina. Debemos anticipar una suspensión de larga duración, centrar los esfuerzos en tratar de asegurar la continuidad formativa y la equidad, cambiar el énfasis de la evaluación hacia la evaluación formativa para el aprendizaje, generar mecanismos de apoyo a estudiantes en desventaja, documentar los cambios introducidos y sus impactos, aprender de nuestros errores y escalar la digitalización, hibridación y el aprendizaje ubicuo; así como realizar una profunda reflexión sobre la renovación de nuestros modelos educativos. "La pregunta del millón es si, en el supuesto de la continuidad de las actividades de enseñanza, los estudiantes conseguirán lograr los objetivos de aprendizaje diseñados para el curso";46 lo que conduce a explorar mecanismos innovadores de enseñanza, aprendizaje y evaluación en los estudiantes de profesiones de la salud, ya que tenemos que reconocer que antes de la pandemia, la situación educativa en nuestro sistema de salud y educativa tenía, por decirlo suavemente, varias áreas de oportunidad.

La pandemia obligó a transitar de forma abrupta a la educación a distancia, proceso que afectó la educación programada de todos los estudiantes de medicina y enfermería, al cerrar las universidades por el confinamiento en casa, retirar a estudiantes de los campos clínicos y transformar la enseñanza habitual en el sistema de salud en un escenario dedicado primordialmente a la atención de pacientes con 
COVID-19. La migración súbita al uso de la educación a distancia puso en jaque las habilidades docentes de los profesores y complicó fuertemente los de por sí amplios retos administrativos de las facultades y escuelas de ciencias de la salud, ya que además de migrar a una modalidad a la que no estábamos acostumbrados, tuvimos que hacer malabares curriculares y de evaluación para tratar de cumplir en tiempo y forma con los planes y programas calendarizados en el ciclo escolar.

Es importante tener claro que la educación que vivimos en los meses de marzo en adelante de 2020 no fue "educación en línea" en toda la extensión de la palabra, sino "educación remota de emergencia", que no es lo mismo. ${ }^{47}$ En contraste a las experiencias educativas planeadas desde un principio para ser en línea, la educación remota de emergencia es un cambio temporal a una modalidad instruccional alterna, debido a la crisis ocasionada por la pandemia. Implica el uso de soluciones totalmente a distancia, que se enseñarían como cursos cara a cara o híbridos, y que se supone regresarán a su formato original cuando cese la emergencia. La importancia de esta diferencia radica en que debemos tener claro que la respuesta a la emergencia tuvo mucho de improvisación y estrategias no ideales, a diferencia de lo que implica la educación en línea de calidad, es decir, actividades en línea planeadas con anticipación y pedagógicamente sólidas, en ambientes virtuales de aprendizaje profesionalmente diseñados y con adecuada formación docente para utilizar las tecnologías modernas en la enseñanza y evaluación a distancia.

Si bien se respondió de manera eficiente a la urgencia educativa a través de un enorme esfuerzo, lo que se proporcionó a los educandos dista de ser lo ideal y se generaron varios vacíos en la enseñanza de habilidades clínicas, psicomotrices y prácticas de campo que eventualmente deben ser remediados. Lo anterior implica que en los meses y años próximos debemos incorporar de manera apropiada las diversas modalidades y modelos de educación en línea, presencial y mixta/híbrida, con estrategias de formación docente y desarrollo curricular que utilicen las buenas prácticas educativas. Para ello es necesario repensar nuestros planes y programas de estudio, los métodos de enseñanza y evaluación del aprendizaje; así como colaborar con colegas de diversas escuelas y facultades para desarrollar estrategias educativas que respondan al descomunal reto que enfrentamos. ${ }^{48}$

Es necesario reflexionar que la educación en las profesiones de la salud tiene impacto en el largo plazo, por lo que sus resultados en la atención clínica de los enfermos y las poblaciones no son fácilmente visibles en el corto plazo, así que habrá que, sin dejar de atender lo urgente, preocuparse y ocuparse por lo importante en el mediano y largo plazo. Es sorprendente lo rápido que los docentes y estudiantes migramos a experiencias educativas en línea; las escuelas y facultades que tienen centros de simulación incrementaron sus actividades y se ha hecho visible la importancia de la simulación virtual y el desarrollo de modelos de "realidad extendida". Las amplias discusiones sobre los roles de los estudiantes en el sistema de salud han generado apasionados y acalorados debates, con fuertes cuestionamientos éticos y de profesionalismo de diversos sectores de la sociedad. En palabras de Kevin Eva, editor de la revista Medical Education, vivimos "días extraños". ${ }^{49}$

Uno de los efectos colaterales adversos de vivir una educación remota de emergencia fue que varios docentes desarrollaron lo que algunos autores Ilaman "covido-pedago-fobia", un rechazo a esta modalidad educativa, ya que al ser su primera experiencia con educación a distancia en este contexto tan difícil y desagradable, es natural que no deseen continuar a largo plazo con este tipo de enseñanza. ${ }^{50}$ Es importante que los profesionales de la educación en las universidades, acompañados del personal experto en educación en línea y tecnologías de información y comunicación, participen de manera intensa en actividades de formación docente para capacitar a los profesores en estas herramientas y diseñen actividades educativas que tomen lo mejor de los dos mundos, el físico cara a cara y el digital a distancia. Debemos acostumbrarnos a vivir con la tecnología no solo en la vida diaria y la práctica de la medicina, sino también en las actividades docentes e ir aprendiendo sobre la marcha de nuestros errores. La educación en línea estaba "guardada", en esa percepción social inapropiada de que es algo de menor calidad o solo para las personas que no pueden asistir a actividades presenciales; ahora está en primera fila y en el centro del escenario del mundo educativo. Es fundamental utilizar la mejor evidencia educativa publicada sobre el tema, generar conocimiento original propio en los contextos locales y avanzar en el desarrollo de recursos y actividades que logren generar entusiasmo en docentes y estudiantes.

Se realizan actividades que creíamos imposibles, tanto clínica como educativamente, en parte por barreras de percepción. En los últimos meses hemos tenido muchas sesiones educativas a distancia con 
nuestros alumnos y hemos participado en infinidad de juntas, sesiones, seminarios web y otras actividades en las que anteriormente utilizábamos mucho tiempo en desplazarnos, encontrar estacionamiento y otros elementos logísticamente complicados; ahora estamos acostumbrados a transitar de una manera bastante fluida entre un espacio virtual y otro, incluidos eventos internacionales y discusiones con colegas en otras partes del mundo. La telemedicina y telesalud también están siendo reconocidas como elementos indispensable en el sistema de salud y el rol de la informática biomédica en la formación de los médicos y especialistas, así como en la práctica clínica, está recuperando espacios importantes. ${ }^{51}$

No hay un consenso internacional absoluto en cuanto a una definición formal de innovación educativa, aunque los siguientes elementos son comunes a la mayoría: se trata de un proceso, debe generar cambio positivo y transformacional, requiere creatividad y motivación de los diferentes actores del acto educativo. ${ }^{52}$ Lo que está ocurriendo a nivel global en la práctica y enseñanza de la medicina debe motivarnos a incorporar innovaciones en la formación de los profesionales de la salud, como el uso de big data y analítica del aprendizaje, el aula invertida (flipped classroom), los cursos masivos abiertos en línea (MOOC, massive online open courses); el uso de gamificación, realidad extendida, Pecha Kucha, entre muchos otros. Es trillado decir que toda crisis es una oportunidad y que lo que no te mata te fortalece, pero en el momento actual de crisis crónica ocasionado por la pandemia sinceramente debemos decir "si no es ahora, ¿entonces cuándo?".

\section{Conflicto de intereses}

Los autores declaran no tener conflicto de interés alguno.

\section{Financiamiento}

Los autores no recibieron patrocinio para llevar a cabo este artículo.

\section{Bibliografía}

1. Freidson E. Profession of medicine. Chicago; III.: The University of Chicago Press; 1988

2. Montori V. Why we revolt: A patient revolution for careful and kind care. Rochester: The Patient Revolution; 2017.

3. Childs B. Genetic medicine: A logic of disease. Baltimore, MD: Johns Hopkins University Press; 2003.

4. Collins $F$, Varmus $H$. A new initiative on precision medicine. $N$ Engl J Med. 2015;372(9):793-795.
5. Berwick D. Eating soup with a fork [Youtube]. XIX National Institute for Healthcare Improvement Forum; 2007. Disponible en: https://www.youtube.com/watch? $\mathrm{v}=\mathrm{dT}$ Tbq9 $\mathrm{cpH} 3 \mathrm{BQ}$

6. Annett J, Stanton N. Task analysis. London: Taylor and Francis; 2000

7. Fletcher S. Analysing competence. Sterling, Va.: Kogan Page; 1997

8. Knigth P. Complexity and curriculum: a process approach to curriculum-making. Teaching in Higher Education. 2001;6(3):369-381.

9. Liebmann R, Costa A. Envisioning process as content. Moorabbin, Vic.: Hawker Brownlow Education; 2005

10. González-Flores P, Luna-de la Luz V. La transformación de la educación médica en el último siglo: innovaciones curriculares y didácticas (parte 1). Invest Educ Med. [Internet]. 2019;8(30):95-109.

11. Morn J, Briscoe G, Peglow S. Current technology in advancing medical education: perspectives for learning and providing care. Acad Psychiatry 2018:42(6):796-799.

12. Chandra A, Nongkynrih B, Gupta SK. Role of smartphone technology in medical education in India. Indian J Community Fam Med 2019;5(2): 103-107.

13. Cheston Ch, Flickinger T, Chisolm M. Social media use in medical education: a systematic review. Acad Med. 2013;88(6):893-901.

14. Masters K, Ellaway R. e-Learning in medical education Guide 32 Part 2 : technology, management and design. Med Teach. 2008;30(5):474-489.

15. Mozer MC, Wiseheart M, Novikoff TP. Artificial intelligence to support human instruction. Proc Natl Acad Sci U S A. 2019:116(10):3953-3955.

16. Vidal-Ledo MJ. Madruga-González A., Valdés-Santiago D. Inteligencia artificial en la docencia médica. Educ Med Super. 2019;33(3):e1970. Disponible en: http://scielo.sld.cu/scielo.php?script=sci_arttext and pi$\mathrm{d}=$ S0864-21412019000300014

17. Hassall Ch, Lewis D. Institutional and technological barriers to the use of open educational resources (OERs) in physiology and medical education. Adv Physiol Educ. 2017:41(1):77-81

18. Gorbanev I, Agudelo-Londoño S, González R, Cortés A, et al. A systematic review of serious games in medical education: quality of evidence and pedagogical strategy. Med Educ Online. 2018;23(1):1438718.

19. Margalef-García L, Arenas M. ¿Qué entendemos por innovación educativa? A próposito del desarrollo curricular. Perspectiva Educacional. 2006; $47: 13-31$.

20. Elmore K. Restructuring Schools. The next generation of educational reform. San Francisco: Jossey-Bass: 1990.

21. Beltrán-Llavador F. Las determinaciones y el cambio del curriculum. En: Angulo F, Blanco. N. Teoría y desarrollo del curriculum. España: Aljibe; 1994

22. Elliott J. The struggle to redefine the relationship between "knowledge" and "action" in the academy: some reflections on action research. Educar. 2004;34:11-26.

23. Bosh OA. De Flexner a Bolonia. Educ Med. 2010;13(4):193-195.

24. San Martín V. La formación en competencias: el desafío de la educación superior en Iberoamérica. Rev lberoamer Educ. 2002·29(1):1-8.

25. González-Flores P, Luna-de la Luz V. La transformación de la educación médica en el último siglo: innovaciones curriculares y didácticas. Invest Educ Med. 2020;9(35).

26. Díaz-Barriga F. Desarrollo del currículo e innovación: modelos e investigación en los noventa. Perfiles Educativos. 2005;27(107).

27. Frenk J, Chen L, Bhutta ZA, Cohen J, Crisp N, Evans T, et al. Health professionals for a new century: transforming education to strengthen health systems in an interdependent world. Lancet. 2010; 376(9756):1923-58

28. López C, Heredia Y. Marco de referencia para la evaluación de proyectos de innovación educativa. Guía de aplicación. México: Instituto Tecnológico y de Estudios Superiores de Monterrey; 2017.

29. Noila-Domenjó M. La evaluación en educación médica. Principios básicos. Educ Med. 2009;12(4):223-229.

30. Maroto-Marín O. Evaluación de los aprendizajes en escenarios clínicos: ¿qué evaluar y por qué? Rev Educ. 2017;41(1):1-18

31. Abreu L.F. et al. Perfil por competencias del médico general mexicano. México; AMFEM; 2008.

32. Hamui-Sutton A, Durán-Pérez VD, García-Téllez SE, Vives-Varela T, Millán-Hernández M, Gutiérrez-Barreto SE. Avances del Modelo Educativo para Desarrollar Actividades Profesionales Confiables (MEDAPROC). Educ Med.2018;19(5):294-300.

33. Hamui-Sutton A, Vives-Varela T, Durán-Pérez VD, García-Téllez SE, Gutiérrez-Barreto S, Millán-Hernández M. Mapeo de una propuesta curricular integral basada en la EPA para UME. MedEdPublish. 2019;8(3):37.

34. Ten Cate O. Entrustability of professional activities and competency-based training. Med Educ. 2005;39(12):1176-7.

35. Schiekirka S, Feufel M.A, Herrmann-Lingen Ch, Raupach T. Evaluation in medical education: a topical review of target parameters, data collection tolos and confounding factors. Ger Med Sci. 2015:13:1-19.

36. Gil ER, Claramunt EFI, Yago JMC, Salas J, López Al, Pueyo CB, et al. Primeras experiencias en evaluación de la competencia clínica de los médicos de familia de Catalunya. Atencion Primaria. 2001;28(2):105-109. 
37. Sociedad Española de Educación Médica. Declaración del Lazareto de Mahón: evaluación de las competencias profesionales en el pregrado. Educación Médica. 2004;7(4):103-105.

38. Salomón-Cruz J, Castillo-Orueta ML, De la Cruz GC. Evaluación en la enseñanza de la Medicina en una institución pública. Perspectivas Docentes. 2015;59:5-12

39. Aungst TD, Clauson KA, Misra S, Lewis TL, Husain I. How to identify, assess and utilise mobile medical applications in clinical practice. Int $J$ Clin Pract. 2014;68(2):155-162.

40. Boushehri E, Soltani Arabshahi K, Monajemi A. Clinical reasoning assessment through medical expertise theories past, present and future directions. Med J Islam Repub Iran. 2015;29:222.

41. Murillo-Sancho G. El portafolio como instrumento clave para la evaluación en educación superior. Rev Actualidades Investigativas en Educación [Internet]. 2012;1(12): 1-23. Disponible en: https://revistas.ucr.ac.cr/ index.php/aie/article/view/10266/18138

42. Thiessen N, Fischer MR, Huwndlek S. Assessment methods in medica specialist assessments in the DACH region. Overview, critical examination and recommendations for further development. GMS J Med Educ. 2019;36(6):Doc78.

43. Martínez A, Trejo JA. ¿Cómo realizar un ECOE? Inv Educ Med 2018;7(28):98-107

44. Hopkins L, Hampton BS, Abbott JF, Buery-Joyner SD, Craig LB Dalrymple JL, et al. To the point: medical education, technology, and the millennial learner. Am J Obstet Gynecol. 2018;218(2):188-192.
45. De Leeuw R, De Soet A, Vandel Horst S, Walsh K, Westerman M, Scheele F. How we evaluate postgraduate medical e-learning: systematic review. JMIR Med Educ. 2019;5(1):e13128.

46. IESALC, UNESCO. COVID-19 y educación superior: de los efectos inmediatos al día después [Internet]; 2020. Disponible en: http://www.iesalc.unesco.org/wp-content/uploads/2020/04/COVID-19-060420-ES-2. pdf

47. Hodges C, Moore S, Lockee B, Trust T, Bond A. The difference between emergency remote teaching and online learning. EDUCASE Rev. 2020 Disponible en: https://er.educause.edu/articles/2020/3/the-difference-between-emergency-remote-teaching-and-online-learning

48. Sánchez-Mendiola M. La educación médica y la pandemia: ¿aislarse 0 colaborar? Invest Educ Med. 2020;9(35):5-7.

49. Eva KW. Strange days. Med Educ. 2020;54(6):492-493.

50. Eachempati $P$, Ramnarayan K. Covido-pedago-phobia. Med Educ. 2020;54(8):678-680.

51. Sánchez-Mendiola M, Martínez-Franco A, Rosales-Vega A, et al. Development and implementation of a biomedical informatics course for medical students: challenges of a large-scale blended-learning program. J Am Med Inform Assoc. 2013;20(2):381-387.

52. Sánchez-Mendiola M, Escamilla-de los Santos J, Sánchez-Saldaña M. ¿Qué es la innovación en educación superior? Reflexiones académicas sobre la innovación educativa. En: Sánchez-Mendiola M, Escamilla-de los Santos J, editores. Perspectivas de la innovación educativa en universidades de México: experiencias y reflexiones de la RIE 360. México: Imagia; 2018. pp. 19-41. 